\title{
Heartbeat: commuting and cardiovascular health
}

Patients and the general public are increasingly aware of the benefits of physical activity in reducing the risk of cardiovascular disease (CVD). Yet, many patients (and physicians) find it difficult to fit a dedicated exercise programme into their busy daily schedule. It has been unclear whether increased physical activity related to commuting or transport in general, has the same benefit as longer blocks of leisure time physical activity. Indeed, a recent article in Heart found that occupational physical activity does not have the same benefit as sports physical activity. ${ }^{1}$ Does this apparent paradox apply to other types of physical activity as well? Or is any activity better than none?

In this issue of Heart, Panter and colleagues $^{2}$ used data from the UK Biobank database, with almost 360 thousand participants, found that regular commuters who use active modes of transport, compared with those who travel exclusively by car, have a lower risk of both incident (HR 0.89, $95 \%$ CI 0.79 to 1.00 ) and fatal (HR 0.70, 95\% CI 0.51 to 0.95 ) CVD (figure 1). In addition, use of active modes of transport for other activities (not simply going to and from work) was associated with an even lower risk of fatal CVD (HR 0.57, 95\% CI 0.39 to 0.85 ).

Readers should listen to Dr. Jenna Panter discussing these results in more detail in a podcast with Dr. James Rudd (https://soundcloud.com/bmjpodcasts/commuting-and-your-cardiovascular-health? in = bmjpodcasts/sets/ heart-podcast).

In the accompanying editorial, Mohanty ${ }^{3}$ discusses the strengths and limitations of this study, concluding that "the study by Panter et $a l^{2}$ provides the best evidence to date that active (vs passive) non-commute travel is associated with better health outcomes, including among individuals who do not regularly commute. This is an important finding, as many individuals may work remotely, do not work, or only commute occasionally and need additional strategies (eg, active non-commute travel) to incorporate regular physical activity into their routines.' She goes on to ask: "Can the

Correspondence to Professor Catherine M Otto, Division of Cardiology, University of Washington, Seattle,WA 98195, USA; cmotto@uw.edu

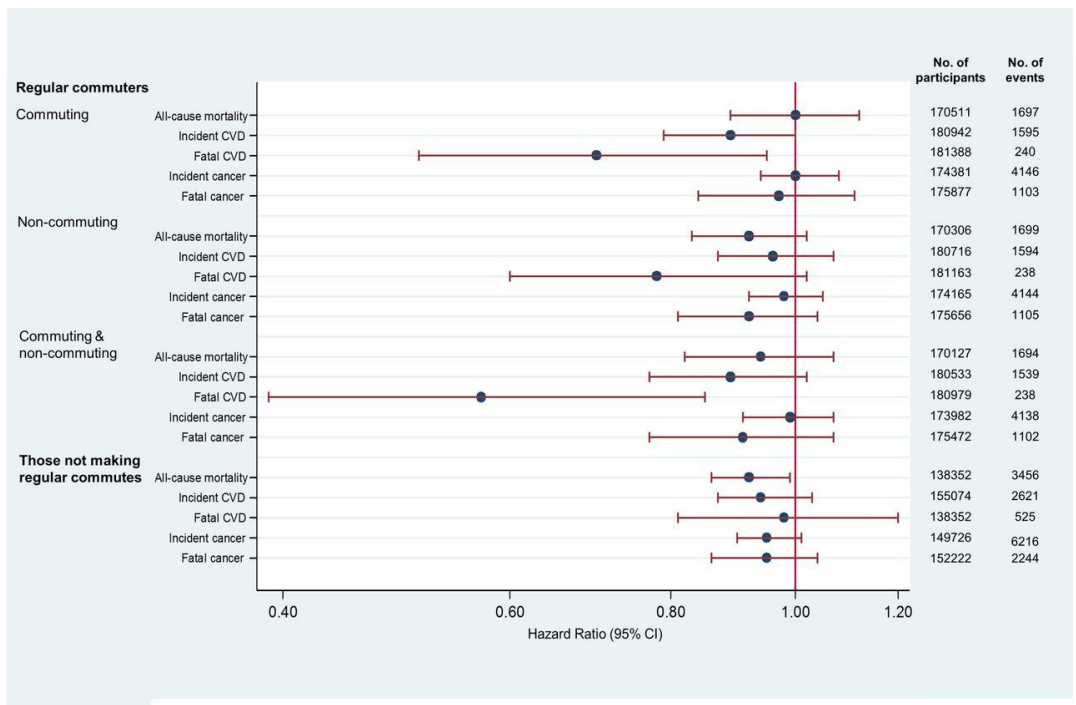

Figure 1 Maximally adjusted HRs for more active patterns of travel (compared with exclusive car use), and all-cause mortality, incident and fatal cardiovascular disease (CVD) and cancer for regular commuters and those not making regular commutes. The HR values for commuting and non-commuting travel are for commuters who use more active patterns of travel at least some of the time relative to commuters who rely exclusively on the car for both commuting and noncommuting travel.

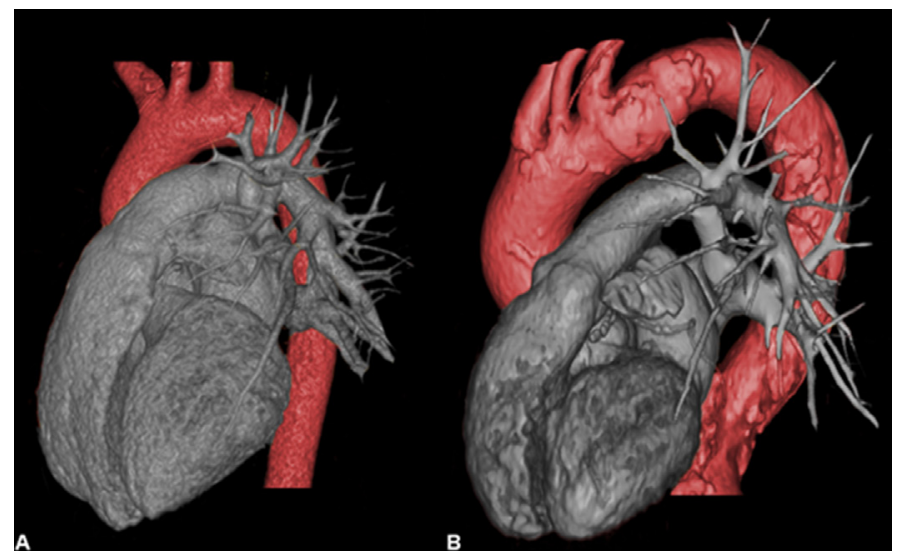

Figure 2 Three-dimensional CT models of the aorta. Three-dimensional modelled CT images of the aorta of a female aged 24 years (A) and a female aged 85 years (B). In part A, a type I aorta is displayed, as the apex of the arch is located between the branch vessels. Part B depicts a type II aorta, as the apex has shifted to a position distally of the left subclavian artery.

findings of this study be extended beyond urban UK? Caution may be warranted, especially where sociocultural and physical environment differences exist. Access to safe bike or walking paths, weather/ seasonal patterns and air quality, among others, may result in different findings.'

Aortic dissection is an uncommon but highly mortal, consequence of hypertension. However, our current standards for risk stratification of adults with aortic dilation are suboptimal, with monitoring and intervention recommendations typically based on a single linear diameter of this complex anatomical structure. In a pair of articles, a research group from Maastricht used 3D computed tomographic imaging of the aorta to provide a more nuanced appreciation of the changes in size and shape or the aorta with normal ageing ${ }^{4}$ and in patients at risk 


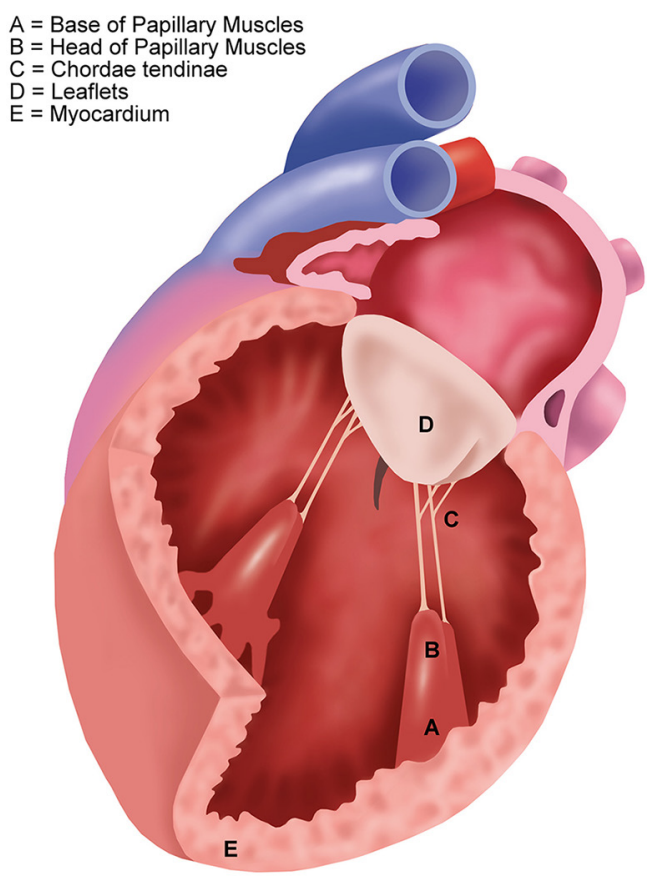

Figure 3 Type IIlb functional mitral regurgitation underlying left ventricular disease which leads to progressive papillary muscle displacement and subsequently increasing leaflet tethering in functional mitral regurgitation. The figure indicates different points of action used in subannular mitral valve reconstruction techniques.

of aortic dissection. ${ }^{5}$ These data show that in normal adults the proximal descending aorta undergoes a $2.5 \mathrm{X}$ increase in length between age 20 and 80 years, with the maximal curvature of the aortic arch moving more distally with age. (figure 2) In a propensity matched analysis of adults with a Type A dissection, compared with controls, the ascending aorta was longer $(78.6 \pm 8.8 \mathrm{~mm}$ vs $68.9 \pm 7.2 \mathrm{~mm}$, $\mathrm{P}<0.001)$ as well as more dilated $(39.4 \pm 5.7$ vs $34.4 \pm 3.2, \mathrm{P}<0.001$ ).

In their editorial, Akin and Nienaber ${ }^{6}$ remind us that "aortic diameter is currently the best-evaluated predictor for aortic dissection with specific 'hinge points' at which rupture or dissection is increasingly likely to occur. These hinge points are set at around $6 \mathrm{~cm}$ for the ascending aorta and $7 \mathrm{~cm}$ for the descending aorta, with current recommendations for preventive treatment when the diameter exceeds $5.5 \mathrm{~cm}$ regardless of location. For patients with Marfan syndrome or other hereditary conditions, bicuspid aortic valve or family history of aortic dissection, the threshold is set lower at $4.5 \mathrm{~cm}$ or $5.0 \mathrm{~cm}$.' However, the International Registry of Aortic Dissection showed that "nearly $60 \%$ of patients with type A dissection had an aortic diameter $<5.5 \mathrm{~cm}$ and almost $40 \%$ had diameters $<5.0 \mathrm{~cm}$, suggesting that aortic diameter alone is an insufficient marker to predict the risk of dissection, and highlighting the need data presented by the Maastricht group, they conclude: "both the dynamic diameter and the length of the aorta play an important role in the pathophysiology of aortic dissection. Nevertheless, it should be emphasised that aortic dissection is triggered by multiple other factors.'

Secondary mitral regurgitation (MR) is a common complication of ischaemic heart disease but intervention to reduce the severity of $\mathrm{MR}$ in these patients remains controversial. (figure 3) In a meta-analysis of over 1000 patients with secondary (mostly ischaemic) MR, surgical sub-annular repair plus ring annuloplasty was associated with a lower rate of recurrent moderate or greater MR compared with those treated with standard surgical annuloplasty alone (OR 0.27, 95\% CI 0.19 to $0.38, \mathrm{P}=0.0001) .^{7}$ Hung, Gammie and Ailawadi ${ }^{8}$ comment that: "Overall, patients with ischaemic MR have significant morbidity and poor prognosis and we need to find innovative, efficacious therapeutic options for these patients. The ideal for better predictors.' Based on the new operation for ischaemic MR is yet to be discovered. It should be a repair (rather than a replacement), result in durable relief of MR and it should be reproducible. We should 'look under the annulus' as this may hold the key for an improved therapy for ischaemic MR.'

The Education in Heart article in this issue discusses interventional management of paravalvular leak, summarising diagnosis and management, including medical therapy, transcatheter closure and surgical correction $^{9}$.

Try the Image Challenge question ${ }^{10}$-what is the next best step in management of this young man with cardiac arrest and unusual coronary angiographic findings?

Competing interests None declared.

Patient consent Not required.

Provenance and peer review Commissioned; internally peer reviewed.

(C) Author(s) (or their employer(s)) 2018. No commercial re-use. See rights and permissions. Published by BMJ.

\section{Check for updates}

To cite Otto CM. Heart 2018;104:1725-1726.

Heart 2018;104:1725-1726.

doi:10.1136/heartjnl-2018-314133

\section{REFERENCES}

1 Ferrario MM, Roncaioli M, Veronesi G, et al. Cohorts Collaborative Study in Northern Italy (CCSNI) Research Group. Differing associations for sport versus occupational physical activity and cardiovascular risk. Heart 2018;104:1165-72.

2 Panter J, Mytton O, Sharp S, et al. Using alternatives to the car and risk of all-cause, cardiovascular and cancer mortality. Heart 2018;104:1749-55.

3 Mohanty AF. Is it time to get out of the car for physical activity benefits? Heart 2018;104:1727-8.

4 Adriaans BP, Heuts S, Gerretsen S, et al. Aortic elongation part l: the normal aortic ageing process. Heart 2018;104:1772-7.

5 Heuts S, Adriaans BP, Gerretsen S, et al. Aortic elongation part II: the risk of acute type $A$ aortic dissection. Heart 2018; 104:1778-82.

6 Akin I, Nienaber CA. Age-dependent aortic elongation: a new predictor for type A aortic dissection? Heart 2018;104:1729-30.

7 Harmel EK, Reichenspurner H, Girdauskas E. Subannular reconstruction in secondary mitral regurgitation: a meta-analysis. Heart 2018:104:1783-90.

8 Hung J, Gammie JS, Ailawadi G. Ideal therapy for secondary mitral regurgitation: should we look under the annulus? Heart 2018;104:1731-2.

9 Eleid M. Interventional management of paravalvular leak. Heart 2018;104:1797-1802.

10 Huang HD, Lombardi WL, Steinberg ZL. Young man presenting with out-of-hospital cardiac arrest. Heart 2018;104:1771. 